\title{
WHITE DWARFS AS OBSERVED AT HIGH SIGNAL TO NOISE
}

\author{
Jesse L. Greenstein \\ Palomar Observatory and Department of Astronomy \\ California Institute of Technology, Pasadena, CA 91125, USA
}

\begin{abstract}
The goal is largely historical, 30 years of instrumental progress in a difficult new field, faint white dwarfs, and some results. High signal-to-noise spectrophotometry at 40-160 $\AA$ resolution revealed the separation between hydrogenand helium-rich atmospheres, and provided a temperature scale from models. The white-dwarf color-luminosity relation proved narrow. Their simple spectra made brute-force averaging possible to 14th magnitude at good photographic resolution. Features as shallow as $5 \%$ and $200 \AA$ wide included $C_{2}$, but in magnetic stars some strong absorptions remain unidentified. Metals are deficient, gravitational diffusion setting the surface composition. The Palomar double CCD spectrograph can now give $S / N \approx 100$ to $17^{m}$. Some polarized white dwarfs have Zeeman triplets in magnetic fields near 20 megagauss. In one, Zeeman components are shifted up to $2000 \AA$ at 300 megagauss. Rotation is small in all white dwarfs, angular momentum mostly lost. Non-LTE cores of $H \alpha, H \beta$ exist and permit improved gravitational redshifts. An evolutionary phenomenon is progressive steepening of the Balmer decrement below $7000 \mathrm{~K}$, cool atmospheres being helium-dominated.
\end{abstract}

\section{INTRODUCTION; EARLY HISTORY OF SPECTROPHOTOMETRY}

I am indeed honored that this Symposium of the IAU is to be dedicated to me, and especially regret that I cannot be physically present. It is even more exciting to see how many new workers and revolutionary topics fill the program. Stellar spectroscopy has gathered new strength from technology, area detectors with high quantum efficiency and massive data handling. For theorists model atmospheres seem no longer tedious and atoms are better understood. While new fields are glamorous (e.g. astroseismology and stellar granulation), others from the 1950's are exciting, like lithium abundances, compositions of globular clusters, heavy-element abundances in population II, the $C, N, O$ ratios and the chemicallypeculiar stars. The Symposium deals with classical and novel problems in the mainstream of astrophysics strengthened by the new possibility of observations with high signal-to-noise ratio, (hereafter $S / N$ ). My paper will emphasize the history of observational data about white dwarf spectra, with current opinions on their physical state. I illustrate mainly white dwarfs with strong magnetic fields; many viewgraphs of typical spectra shown at the Symposium are omitted for economy of space. A few are shown at the end. 
The concept of high $S / N$ seems implausible to those whose experience began with photographic stellar spectroscopy which could not have high $S / N$. The invaluable Utrecht Solar Atlas, had good $S / N$, obtained from high-resolution spectrographs with high-contrast emulsions. Poor $S / N$ was especially characteristic of spectra of the white dwarfs, (hereafter WD), mostly fainter than 14th mag. My own first use of high $S / N$ was in the attempt by Greenstein and Richardson (1951) to observe both ${ }^{7} \mathrm{Li}$ and ${ }^{6} \mathrm{Li}$ in the Sun. Its depletion to $1 \%$ of the terrrestrial value lead us to the hypothesis of its destruction by nuclear reactions after mixing to about two million degrees.

For stars, the Schmidt cameras and Babcock gratings of the Mount Wilson coudé spectrograph gave widened photographic spectra at up to $1.0 \AA \mathrm{mm}^{-1}$. Greenstein (1956a) compares a photographic coudé spectrum of the daylight sky, processed through a linearizing microphotometer, with the Utrecht Solar Atlas. Both have low noise; the lower resolution of the coudé makes weak lines shallow but still shows some lines of Rowland intensity -1 . Oke pioneered spectral scanning at Mount Wilson and later at Palomar; one of his first instruments was a two-channel, high-resolution scanner at the coudé; its output (Oke and Greenstein 1961) gave high $S / N$ on bright stars, slowed by the need to scan.

In that paper van Maanen 2 (EG 5, 0046+05) a cool 13th mag white dwarf is also illustrated. From $4000 \AA$ to the ultraviolet, strong, broadened and blended $\mathrm{Fe} I, \mathrm{Ca} I I, \mathrm{Mg} I$ are seen. The features have low contrast, many too broad to be easily "visible" by inspection. Tracings of 5 Palomar coudé spectra were measured objectively at constant wavelength intervals without smoothing plate grain and then averaged. The resolution is $1.8 \AA$; noise is reduced by brute-force averaging. Reductions were tedious but features $5 \%$ deep were found. Weidemann (1960) analyzed these spectra to show that metals were deficient by factors of $\approx 10^{-4}$. The helium opacity is low; the low metal abundance was the first evidence of how abnormal was the composition of WD's. We now believe that gravitational diffusion separates metals from the helium. With the IUE the star was found to be 19 th mag in the $2800 \AA$ line of $M g I I$.

Brute-force averaging of photographic spectra is particularly effective in giving a "collective" high $S / N$, to detect features spanning many resolution elements. Greenstein and Matthews (1957) used this averaging procedure on spectra of faint WD's; Figure 1 illustrates Wolf 219 (EG 24, 0341+18), with a broad features $10 \%$ deep, $200 \AA$ wide on three spectra. The $4670 \AA$ feature was identified as $C_{2}$ with extreme pressure broadening; a band $3 \%$ deep at $4355 \AA$ is also $C_{2}$. Later the $(0,0)$ band at $\lambda 5165$ was found. The atmosphere is dominantly helium and the ultraviolet resonance lines of $C I$ were found with $I U E$ in carbon-rich WD's, (type DQ). Using the Kitt Peak Image-Intensified Dissector Scanner and helium-dominated models, Wegner and Yackovich (1984) studied abundances in DQ's from $C_{2}$. They found the $C / H e$ ratio to be $10^{-2}-10^{-7}$ with $H, N, O$ in low abundance over a range of $T_{\text {eff }}$ where carbon is mixed to the surface from the degenerate core.

Figure 2 here shows the extraordinary object Grw $+70^{\circ} 8247$ (EG 129, $1900+70$ ), with mean profiles from five spectra (Greenstein $1956 \mathrm{~b}$, Greenstein and Matthews 1957). The quality is comparable to the less noisy CCD spectrum illustrated in Greenstein, Henry and O'Connell (1985) at $12 \AA$ resolution and 20000 counts per pixel. Only $\lambda 4135$ was "visible" in Minkowski's original discovery in 1938. This resisted identification; in $1984 \mathrm{I}$ identified $\lambda 1347$ on the $I U E$ spectrum as the Lyman- $\alpha$ Zeeman component, 1s 0 - 2p-1. Then 10 Zeeman components of $\mathrm{H} \alpha, \mathrm{H} \beta$ and $\mathrm{H} \gamma$ were successfully identified, from quantum-mechanical computa- 
tions of hydrogen in strong magnetic fields. Figure 3 of $\lambda 4135$ shows the fit to $2 \mathrm{~s} 0$ - $4 \mathrm{fO}$ of $\mathrm{H} \beta$ (Greenstein, Henry and O'Connell 1985; Angel, Liebert and Stockman 1985).

The 1957 mean of five spectra of $\mathrm{HZ} 29$ showed doubled lines of $\mathrm{HeI}$ averaging $5 \%$ in depth and never exceeding $10 \%$. This star is a helium-rich masstransfer variable, AM CVn (EG 91, 1232+37); it probably consists of two white dwarfs that will ultimately merge, and according to theorists, produce a supernova of type I. The line profiles did not move or change. A similar interacting pair of helium white dwarfs is G61-29, showing broad emission lines of $\mathrm{HeI}$.

I mention these objects because the spectra have not been substantially surpassed in published recent papers using electro-optical sensors. Some numbers and a critique concerning photographic spectrophotometry of faint stars may be useful. A typical WD spectrum at the 200-inch prime-focus, widened to $0.3 \mathrm{~mm}$ at $180 \AA \mathrm{mm}^{-1}$, took 60 minutes at 15 th mag. What is the noise in such spectra? The projected slit included $\approx 20$ developed plate grains. The number of photons required by a single plate grain was $\approx 50$, so the photon noise (from the required 1000 photons) is not serious. Plate grains are essentially opaque and their summed response is non-linear; the small number of grains per resolution element is the noise on the microphotometer tracing. For most purposes, the resolution of $\approx 5$ $\AA$ was higher than needed, because the WD's stark- or pressure-broadened lines are wider. Plates are more uniform in response than are pixels on a CCD, so flat-fielding is unnecessary. In summary, careful photographic spectrophotometry could detect subtle WD features to a depth of about $3 \%$. None of these objects needed sky subtraction, being photon-starved by the inefficiency of the plates, rather than sky-limited. What we had was "detectability" rather than high $S / N$. Other wavelength regions are more contaminated by airglow emission than the blue, where photographic spectrophotometry was limited to 15 th mag.

A serious problem encountered in pioneering high-dispersion coudé studies of normal stars are worth recall. We were forced to a much effort on the scales of line intensities, which varied between observatories and depended on dispersion. IAU Commissions became interested and involved in an international attempt to provide standard equivalent widths and profiles (Wright et al. 1964) resulted. The optical quality of the older coudé spectrographs was excellent and the photographic calibration careful; scattered light and grating ghosts were investigated. I wonder whether similar attention is paid now to such questions? Solid-state detectors and especially echelle spectrographs in new spectrometers might usefully be intercompared by observations of standard stars.

\section{THE WHITE DWARFS; PROGRESS IN CAPABILITY}

Given the limitations on $S / N$ it is fortunate that the apparent simplicity of most WD spectra is real; problems arising from a background of weak, highly pressurebroadened, invisible lines have not surfaced. The important result of much analysis is that a dichotomy of composition exists. The more numerous group is hydrogendominated (the so-called DA types), the other is helium-dominated (the non-DA's). In the DA's the abundance ratio is $H / H e \geq 10^{3}$; in the non-DA's, $\mathrm{He} / \mathrm{H} \geq 10^{4}$. The DA's have essentially no metals, while the non-DA's sometimes have carbon and metals, but always in abundances far below their solar values; mixed $\mathrm{H}+$ He compositions are rare. In DA's the Balmer lines coalesce beyond H8; pressure broadening destroys the higher states depressing the ionization potential. The Balmer continuum is strong; hydrogen lines and continuum apparently disappear 
at $T \leq 7000 \mathrm{~K}$ (see §III). Among the non-DA's, He II is seen rarely (defining the $\mathrm{D} \overline{\mathrm{O}}$ type) at high temperatures; over a limited range of temperatures, He I is strong, broad and shows forbidden components in DB's. Carbon is present in cooler stars, called type DQ; metals appear weakly in a few WD's, mostly below $12000 \mathrm{~K}$, called DZ's. Both carbon and metals are confined to the non-DA's and result from the low opacity of helium as well as from genuine abundance anomalies at the surface.

Given the relative simplicity of the WD spectra the multichannel spectrophotometer (the MCSP) designed and built by J. B. Oke proved nearly ideal for surveys. The high quantum efficiency of 31 photomultipliers provided simultaneous observation in two apertures, sky, and sky + object, with high accuracy in short times. To illustrate the sensitivity of the MCSP, a DA star GD 356 ( Gr 329, $1653+53$ ) has $m_{V}=15.3$; it gave $\approx 2 \times 10^{4}$ detected photons in 360 seconds at 5400 $\AA$ with $40 \AA$ resolution $(3100 \leq \lambda \leq 5700)$ and $80 \AA$ resolution $(5700 \leq \lambda \leq 10000)$. This visual flux corresponds to 2.7 milliJanskies; I discuss this star in $\S I I I$. By stepping the grating to five wavelengths and switching apertures, the entire spectrum is covered, with redundancy. We obtain $m_{\nu}$ on the Oke AB scale, and $\log f_{\nu}$, at 124 wavelengths, in 60 minutes, most with photon-counting statistics of \pm 0.01 mag. Data are better suited to model-atmosphere fitting than are the broadband photoelectric magnitudes, because of the nearly monochromatic fluxes.

Colors observed with the MCSP for over 300 WD's with $\approx 1-2 \%$ errors are in Greenstein (1984), on the absolute AB79 scale of Oke and Gunn (1983). The correlation of color with luminosity for WD's with modern parallax is excellent (Greenstein 1985). The intrinsic (or cosmic) scatter appears to have a $\sigma$ near \pm 0.25 mag; the resulting spread in radius is only $12 \%$, less if the color- $T_{\text {eff }}$ relation is not unique. Weidemann and collaborators at Kiel have fitted model atmospheres to fluxes observed in both hydrogen and helium WD's. The absolute calibration of $\mathrm{AB}$ is central to the temperature scale; spectroscopic gravities average log $g=+8.0$ (Koester, Schulz and Weidemann 1979), agreeing with those from parallaxes and temperatures. Weidemann and his group specialized in obtaining spectroscopic masses. Predicted and observed gravitational redshifts agree if the core of the WD has $\mu_{e l}=2.0$, i.e. is helium or carbon and the typical mass is near $0.6 \mathrm{M} \odot$ (see $\S 5)$. The MCSP and hydrogen models define the limited range of temperatures in which rapid light variation of the $\mathrm{ZZ}$ Ceti type occurs. These complex oscillations occur in DA's, with $T_{\text {eff }}$ confined to within $\pm 500 \mathrm{~K}$ of $11200 \mathrm{~K}$ (Greenstein 1982), in agreement with the theory of non-radial modes of hydrogen envelopes.

The wide wavelength range covered by the MCSP at good $S / N$ favors detection of broad features, success depending on the smoothness of the heterochromatic calibration. The MCSP revealed broad "waves" in otherwise continuous spectra of WD's with circular and linear polarization. Many of these features remain unidentified. The cool degenerate G240-72 (Gr 372 1748+70) has a dip 2400 $\AA$ wide, reaching nearly $20 \%$ and centered on $5260 \AA$ (Greenstein 1974). GD 229 ( $\mathrm{Gr} \mathrm{333,2010+31)}$ is shown in Figure 4. It is hot and has still unidentified strong dips at wavelengths $4185,5280,7090$ and $8000 \AA$ which are correlated with the wavelength dependence of circular polarization. Greenstein and Boksenberg (1978) counted 5000 photons per pixel at the coude with the IPCS to show that the $4185 \AA$ feature had $\approx 300 \AA$ FWHP, with details of structure unchanged from four years earlier with the MCSP. It may have cyclotron absorption. Liebert et al. (1976) found Zeeman components of $\mathrm{HI}, \mathrm{HeI}$ in Feige 7 ( $\mathrm{Gr}$ 267, 0041-10) shifting as it rotates with in a 2.2 hour period.

A fascinating problem remains unresolved for the coolest degenerates. 
What explains a spectrum without any lines? In early work, I called WD's with no lines deeper than $5 \%$ type DC. Helium opacity is so low in a hydrogen-free atmosphere that unsaturated resonance lines should be visible, if even only a trace of a metal exists. Can lines be pressure-broadened into invisibility by the gas pressure, which exceeds 100 atmospheres? Can all bound states except the ground state be destroyed? Laboratory gases at such pressures have extraordinary spectra with broad asymmetric "waves", sometimes located near resonance lines. Quasimolecules and polymers are formed. While I doubt that the apparent lack of lines in cool white-dwarfs is caused only by pressure broadening, the observational evidence is still marginal. The only attempt was a study (Greenstein 1979) of the smoothness of the mean fluxes in nine faint, red DC degenerates with $M_{V} \geq 15$. The residuals from the mean flux and its quadratic representation were evaluated from 8000 to $4200 \AA$. They had the remarkably small scatter of $2 \sigma \leq 0.02 \mathrm{mag}$, except from 4000 to $3500 \AA$ where they are rough and depressed by 0.05 mag. Unfortunately the calibration of sdG's in this region is uncertain at that level, because of metallic lines. The search for "invisible" lines remains an important problem in absolute calibration for high $S / N$ detectors.

\section{HIGHER RESOLUTION AND S/N; COMPOSITION; ZEEMAN EFFECT}

That higher $S / N$ would be important for study of physical processes in WD's was demonstrated by Gary Wegner, with Kitt Peak National Observatory spectrometers. He showed that many apparently line-free, so-called DC's actually had weak lines, sometimes hydrogen in the cool DA's, or $C_{2}$ bands in the DQ's. Wegner and Yackovich (1982) and Wegner (1983) observed DC's at $17 \AA$ resolution; of 46 WD's originally called DC, 18 remained line free, 4 had $\mathrm{H} \alpha, 8$ had $H e I, 9$ had $C_{2}, 1$ had $C I$, and 6 remained uncertain. The improved $S / N$ revealed helium-rich atmospheres, and weak hydrogen, given the extra effort. Wegner's results broadened our ideas about the carbon-rich WD's. Wegner and Yackovich (1984) found the $\mathrm{H} / \mathrm{He}$ ratio low and the $\mathrm{C} / \mathrm{He}$ ratio to range over $10^{5}$ critically dependent on temperature. Convection occuring over a limited range of $T_{\text {eff }}$ dredges carbon up from the degenerate core.

I had only one chance to use the Oke-Gunn double spectrograph at the cassegrain focus of the 200-inch, a genuinely high $S / N$ instrument for moderate resolution on stars. It is provided with two excellent CCD's of high quantum efficiency, flat fielding and sky subtraction. Saturation is near 30000 detected photons per pixel; short exposures reach 17 th mag. I observed 140 white dwarfs in eight nights. In 1982, after my retirement, I used it with low-dispersion gratings to cover the entire WD spectrum; lines of equivalent width below $1 \AA$ are just detectable.

From these CCD spectra a major new result (Greenstein 1986) was that the fraction of WD's whose atmosphere contains hydrogen lines falls rapidly at $T_{\text {eff }} \leq 6000 \mathrm{~K}$. The Balmer decrement changes; $80 \%$ of WD's with $T_{\text {eff }} \geq$ $7000 \overline{\mathrm{K}}$ show $W(H \alpha) / W(H \beta) \approx 0.8$, the normal ratio. Their atmospheres are dominantly hydrogen. This fraction drops below $20 \%$ among cooler WD's, which are dominantly helium. $\mathrm{H} \alpha$ finally disappears at $4500 \mathrm{~K}$ (at $M_{V} \geq+15$ ) but remains surprisingly sharp. Figure 5 shows LHS 5023 ( $\mathrm{Gr} 462,0102+\overline{2} 1 \mathrm{~A}$ ), which is among the coolest known degenerates. The Figure also graphically illustrates the power of the CCD spectrograph and the virtue of even moderately high $S / N$. The star is near 18th magnitude, the observing time was one hour, the line is $4 \%$ deep and visible in at most 4-6 pixels. This type of $\mathrm{H} \alpha$ is not present in all cool DC's 
and may be enhanced by resonance broadening or non-LTE effects, stronger than expected from pure hydrogen models. Greenstein (1986) illustrates a mean profile formed to improve $S / N$ in three other faint red degenerates near $4500 \mathrm{~K} ; \mathrm{H} \alpha$ is $3 \%$ deep and visible in several pixels, but no significant absorption is visible at $\mathrm{H} \beta$. In contrast, $\mathrm{H} \beta$, (measured in a second-order grating spectrum at better resolution) decreases as predicted by the same models. High $S / N$ on faint red degenerates is an important and still difficult task for large telescopes. The observed ratio of $W(H \alpha) / W(H \beta)$ increases to above 2 at $6000 \mathrm{~K}$, as $\mathrm{H} \beta$ fades into invisibility faster than $\mathrm{H} \alpha$. The most probable explanation comes from computations of atmospheres containing mixtures of helium and hydrogen. An abnormal Balmer decrement in WD's was first explained by Wehrse (1977) before it was observed. Liebert and Wehrse (1983) computed models with different $\mathrm{He} / \mathrm{H}$ abundance ratios, for a star of $7400 \mathrm{~K}$, much hotter than those I have observed. As gas pressure is increased by increasing the $\mathrm{He} / \mathrm{H}$ ratio both Balmer lines strengthen. Even with $1 \%$ hydrogen $\mathrm{H} \alpha$ remains as strong as in pure hydrogen, but $\mathrm{H} \beta$, formed at large optical depth, broadens into invisibility. Calculations of models for cool stars, by Wehrse and by the Montreal group (Fontaine, Wesemael and collaborators) were reported at the IAU Colloquium No. 95 on Faint Blue Stars in Tucson, June 1987. The deduction that the coolest degenerates have helium-dominated atmospheres is quit important in interpreting the balance between convection, gravitational diffusion and possible accretion.

\section{HIGH $S / N$ IN SOME MAGNETIC STARS}

The DA star GD 356 was used in §II as a quantitative illustration of MCSP sensitivity. Because it had an apparently "rough" MCSP spectrum, it was re-observed at $\mathrm{H} \alpha$ and $\mathrm{H} \beta$ with the Oke-Gunn double CCD spectrograph, at $6 \AA$ and $4 \AA$ per pixel. The resolution is degraded to about 2 pixels by the large apertures but the $S / N \approx 1 \%$. Greenstein and McCarthy (1985) found the $\mathrm{H} \alpha$ structure to be a Zeeman emission triplet which is fitted by a pole-on magnetic dipole field of 20 megagauss; the $\mathrm{H} \beta$ emission is weaker and distorted by the quadratic Zeeman effect. The absorption Zeeman triplet was found in the polarized, cool degenerate G 99-47 (Gr 290, 0553+05). Neither a dipole magnetic field of 15 (pole-on) or 27 megagauss (equator-on) fits the observations as well as a tangled field of 14 megagauss with a gaussian distribution of the scalar field, with $\sigma \approx 1.4$ megagauss. It may seem to be a non-physical model; no polarization has been observed in GD 356 , so a tangled magnetic field may have some merit.

In the strongly magnetic star Grw $+70^{\circ} 8247$, the CCD spectra show details of the $\lambda 4135$ line (Greenstein, Henry and O'Connell 1985). This 2s0 - 4f0 component of $\mathrm{H} \beta$ is not forbidden in a strong magnetic field. Various values of the dipole magnetic field, with assumed tilts were tried; the noise level is low, and the fit is fairly good with a nearly pole-on field of $250-300$ megagauss, as illustrated in Figure 3. The strongest line in the red is $2 \mathrm{~s} 0-3 \mathrm{p} 0$ of $\mathrm{H} \alpha$ at $5910 \AA$, best fitted with 300 megagauss field. A strong $\mathrm{H} \alpha$ component exists at $8500 \AA$ which should persist at even higher magnetic field.

\section{ROTATION. GRAVITATIONAL REDSHIFT}

The photon yield for WD's was improved over the photographic plate by the imageintensifier tube; it permitted Greenstein and Trimble (1972) to see sharp absorption cores in $\mathrm{H} \alpha$ and $\mathrm{H} \beta$. The sharp core occurs over a limited range of $T_{\text {eff }}$. With 
resolution of $1.1 \AA$ our estimate was that the rotation $v \sin i \leq 30 \mathrm{~km} \mathrm{~s}^{-1}$. Greenstein and Peterson (1973) confirmed the existence of the sharp, deep cores and advanced the non-LTE explanation, as did Shipman. Hydrogen atoms at small $\tau_{\text {cont }}$ and therefor at low gas pressure see a depressed radiation field $\pm 50 \AA$ from the line center, at a low boundary temperature. Low rotation means that about 99 $\%$ of the angular momentum has been lost as the red giant lost $80 \%$ of its mass. The typical main-sequence parent is a late $\approx 4 \mathrm{M}_{\odot}$ ) B star, rotating at $60 \mathrm{~km} \mathrm{~s}^{-1}$. When it shrinks to the WD radius of $0.01 \mathrm{R} \odot$ it should rotate at $10^{4} \mathrm{~km} \mathrm{~s}^{-1}$, were its angular momentum conserved. Pilachowski and Milkey $(1984,1987)$ with much improved resolution at the Kitt Peak echelle spectrograph beautifully confirmed the slow rotation of DA's. In their 1987 paper, they have good data for 15 stars in the most favorable temperature range for non-LTE cores. They strengthen the limit to rotation (which averages $\approx 30 \mathrm{~km} \mathrm{~s}^{-1}$ ); rotation "is indistinguishable from zero in 10 stars". The highest $v \sin i=60 \pm 10 \mathrm{~km} \mathrm{~s}^{-1}$. Most of the specific angular momentum of the parent star has been lost during the AGB evolution. Cyclic variation in circular polarization showed the magnetic WD's to be even slower rotators than the non-magnetic WD's. I had observed a sharp core in 40 Eri B (EG 33 0413-07) with the Boksenberg IPCS system (0.8 $\AA$ per pixel) at Palomar (Greenstein et al. 1977), for which Pilachowski and Milkey (1987) find rotation of $19 \pm 5 \mathrm{~km} \mathrm{~s}^{-1}$. The non-LTE cores have a steep Balmer decrement, as expected from transition probabilities. The $\mathrm{H} \beta$ core is easily seen, but no core is seen at $\mathrm{H} \gamma$ even with 2500 counts per pixel. Pressure broadening, which strengthens high series members and flattens the decrement, does not operate on the cores.

The sharp core permit velocity measures at relatively high accuracy in a few of the brighter DA's; it should not be pressure-shifted and is objectively measureable. Lines are $6000 \mathrm{~km} \mathrm{~s}^{-1}$ wide; if the apparent centroid of this wide line is used photographic measurement is a delicate art. Trimble and Greenstein (1972) obtained a statistical value of the Einstein gravitational redshift. Velocities corrected for solar motion were averaged to derive the K-term; a few WD's in resolved binaries and clusters gave redshifts. Our mean gravitational redshift was $51 \mathrm{~km} \mathrm{~s}^{-1}$. Wegner (1974) measured redshifts in southern WD's and found the statistical value of $40-50 \mathrm{~km} \mathrm{~s}^{-1}$. Currently, some resolved binaries (DA and $\mathrm{dM}$ ) give individual redshifts on electronic spectra, and therefor reliable $M / R$ determinations. At higher dispersion these may have different sensitivity to pressure shifts; they indicate smaller mean redshift (near $25 \mathrm{~km} \mathrm{~s}^{-1}$ ) and therefor lower masses. Astrometric masses are rare for white dwarfs, e.g. 0.4 and $1.1 \mathrm{M} \odot$ for 40 Eri B and Sirius B. Astrophysical masses use model atmospheres, temperatures and surface gravity and give typical mass near $0.6 \mathrm{M} \odot$. Photographic redshifts suggested about $0.7 \mathrm{M} \odot$; these new, unpublished measures in binaries suggest 0.5 to $0.6 \mathrm{M} \odot$. Improved techniques with such different methods should converge to establish the $M / R$ relation soon.

In conclusion, I again must express my gratitude to those who organized this IAU Symposium and provided to all of us another encounter with modern spectroscopy. In addition to personal friendships we share the good fortune of work in an important and exciting field. 

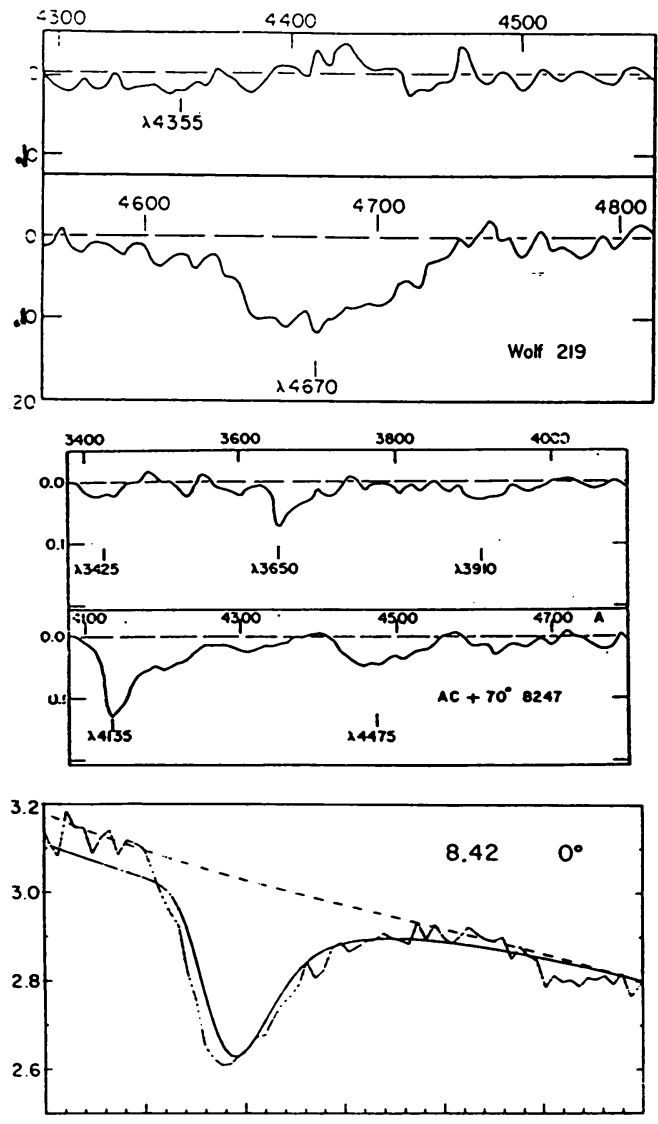

Figure 1. Mean (2) coudé photographic spectra (1957) illustrating the pressurebroadened $C_{2}(1957)$ in a white dwarf Wolf 219; absorption in magnitudes.
Figure 2. Mean (5) coudé photographic spectra of the polarized, magnetic white dwarf Grw $+70^{\circ} 8247$. Absorptions, unidentified until 1984, are Zeeman components of hydrogen.

Figure 3. (Light data points). Single high $S / N$ Palomar CCD spectrum of $\mathrm{Grw}+70^{\circ} 8247$ at the $\lambda 4135$ feature, a component of $\mathrm{H} \beta$. Ticks on wavelength scale are $10 \AA$, flux in milliJanskies. (Solid). Predicted 2s0-4f0 transition of $H \beta$ in a pole-on magnetic field, 260 megagauss.

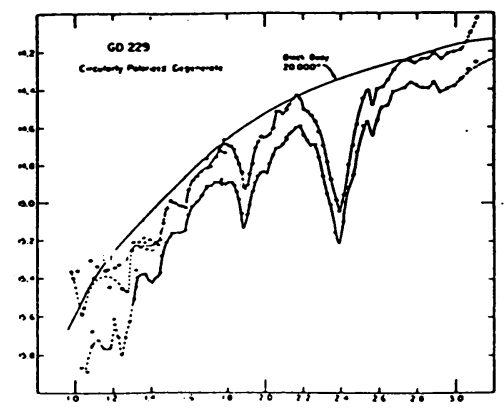

Figure 4. Two multichannel spectra (vertically offset), mag vs. $1 / \lambda$ in $\mu m^{-1}$ of GD 229 , a polarized hot white dwarf with still unidentified features.

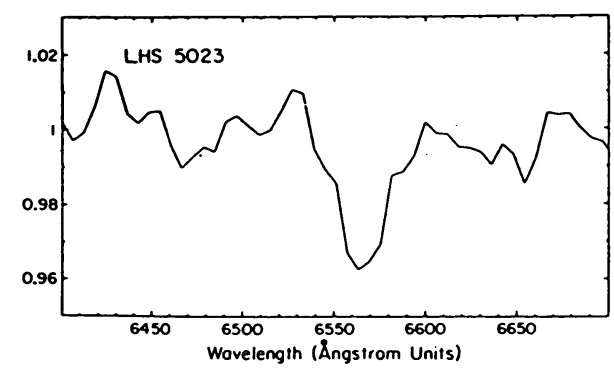

Figure 5. $H \alpha$ at moderate noise in an 18th mag red degenerate LHS 5023 (probably low $\mathrm{H} / \mathrm{He}$ ); Palomar CCD spectrograph, 1 hour. 


\section{REFERENCES}

Angel, J. R. P., Liebert, J. and Stockman, H. S. 1985, Astrophys. J., 292, 260.

Greenstein, J. L. 1956a, Vistas in Astronomy, ed. A. Beer, (Pergamon: London), 2, 1299 .

Greenstein, J. L. 1956b, Proc. 9rd Berkeley Symp. Math. Statistics and Probability, ed. J. Neyman, (Univ. of Calif. Press: Berkeley), 3, 11.

Greenstein, J. L. 1974, Astrophys. J. (Letters), 194, L51.

Grcenstein, J. L. 1979, Astrophys. J., 227, 244.

Greenstein, J. L. 1982, Astrophys. J., 258, 661.

Greenstein, J. L. 1984, Astrophys. J., 276, 602.

Greenstein, J. L. 1985, Pub. Astron. Soc. Pacific, 97, 827.

Greenstein, J. L. 1986, Astrophys. J., 304, 334.

Greenstein, J. L. and Boksenberg, A. 1978, Mon. Notices Roy. Astron. Soc., 185, 823.

Greenstein, J. L. 1985, Henry, R. J. W. and O'Connell, R. F. 1985, Astrophys. J. (Letters), 289, L25.

Greenstein, J. L. and Matthews, M. S. 1957, Astrophys. J., 126, 14.

Greenstein, J. L. and McCarthy, J. K. 1985, Astrophys. J., 289, 732.

Greenstein, J. L. and Peterson, D. M. 1973, Astron. Astrophy., 25, 29.

Greenstein, J. L. and Richardson, R. 1951, Astrophys. J., 113, 536.

Greenstein, J. L. and Trimble, V. 1972, Astrophys. J. (Letters), 175, L1.

Koester, D., Schulz, H. and Weidemann, V. 1979, Astron. Astrophys., 65, 262.

Liebert, J., Angel, J. R. P., Stockman, H. S., Spinrad, H. and Beaver, E. A. 1976, Astrophys. J., 214, 457.

Liebert, J. and Wehrse, R. 1983, Astron. Astrophys., 122, 297.

Oke, J. B. and Greenstein 1961, Astrophys. J., 133, 349.

Oke, J. B. and Gunn, J. E. 1983, Astrophys. J., 266, 713.

Pilachowski, C. A. and Milkey, R. W. 1984, Pub. Astron. Soc. Pacific, 96, 821.

Pilachowski, C. A. and Milkey, R. W. 1987 Pub. Astron. Soc. Pacific, in press.

Trimble, V. and Greenstein, J. L. 1972, Astrophys. J., 177, 441.

Weidemann, V. 1960, Astrophys. J., 131, 638.

Wegner, G. 1983, Astron. J., 88, 1034.

Wegner, G. 1974, Mon. Notices Royal Astron. Soc., 166, 271.

Wegner, G. 1978, Mon. Notices Royal Astron. Soc., 182, 111.

Wegner, G. and Yackovich, F. H. 1982, Astron. J., 87, 155.

Wegner, G. and Yackovich, F. H. 1984, Astrophys. J., 284, 257.

Wehrse, R. 1977, Mem. Soc. Astr. Italiana, 48, 13.

Wright, K. O., Lee, E. K., Jacobson, T. V. and Greenstein, J. L. 1964, Pub. Dom. Astrophys. Obs., 12, 173. 